\title{
Husain-Kuchar model as a constrained BF theory
}

\author{
Merced Montesinos* and Mercedes Velázquę ${ }^{\dagger}$ \\ Centre de Physique Théorique, CNRS Luminy, F-13288 Marseille, France and \\ Departamento de Física, Cinvestav, Instituto Politécnico Nacional 2508, \\ San Pedro Zacatenco, 07360, Gustavo A. Madero, Ciudad de México, México.
}

(Dated: June 20, 2018)

\begin{abstract}
The Husain-Kuchar theory is a four-dimensional background-independent model that has long been viewed as a useful model for addressing several conceptual and technical problems appearing in the quantization of general relativity mainly in the loop quantum gravity approach. The model was defined at Lagrangian level in terms of a $s u(2)$-valued connection one-form $A$ coupled through its curvature to a $s u(2)$-valued one-form field $e$. We address here the problem of writing a Lagrangian formulation for the Husain-Kuchar model as a constrained BF theory motivated by the fact that spin foam models for quantum gravity are related to action principles of the BF type. The Lagrangian action principle for the Husain-Kuchar model reported here differs from a previous one found by Barbero et al in that this description involves a single constrained BF theory rather than two interacting $\mathrm{BF}$ theories. It is, essentially, the Plebanski action with the condition on the trace of the Lagrange multipliers removed. Moreover, it can be stated that the relationship between our BF-like action and the original one for the Husain-Kuchar model is the same relationship that exists between the Plebanski action and the self-dual Palatini action for complex general relativity, first because the solution to the constraint on the two-forms $\Sigma^{i}$ coming from the BF-like action leads to the Husain-Kuchar action, and second because the Hamiltonian analysis of the Husain-Kuchar model is straightforward starting from the BF-like action principle.
\end{abstract}

PACS numbers: 04.60.Ds, 04.20.Cv, 04.20.Fy

It is very well-know that general relativity, expressed in terms of Ashtekar variables [1], and the Husain-Kuchar model [2] are very close to each other. Their similarities are usually appreciated by employing the Hamiltonian form of their corresponding action principles. In particular, these theories have the same phase space variables but the Hamiltonian constraint of general relativity is missing in the Husain-Kuchar model, which is defined by the action principle [2]

$$
S[e, A]=\int_{\mathscr{M}^{4}}\left[\varepsilon_{i j k} e^{j} \wedge e^{k} \wedge F^{i}[A]\right],
$$

where $F^{i}[A]=d A^{i}+\varepsilon^{i}{ }_{j k} A^{j} \wedge A^{k}$ is the curvature of the $s u(2)$-valued connection 1 -form $A=A^{i} J_{i}, e=e^{i} J_{i}$ is a $s u(2)$-valued one-form field, and $J^{i}$ are the generators of $s u(2)$ and satisfy $\left[J_{i}, J_{j}\right]=\varepsilon^{k}{ }_{i j} J_{k}$.

Perhaps one of the best ways to understand the relationship between the self-dual Palatini action 3, 4] and the action principle (10) is to look at the self-dual Palatini action as a constrained Husain-Kuchar model obtained by adding the constraint $e^{i} \wedge F_{i}[A]$ to the action (11) with a Lagrange multiplier 1-form $\lambda$ [5]. On the other hand, the Husain-Kuchar model can also be described by an action principle involving $e^{i}, A^{i}$, and a scalar field $\phi$ enlarging in this way the phase space but introducing an additional scalar constraint which allows it to preserve the three local degrees of freedom of the model [ [6]. Even though these works are very interesting and valuable because they shed light on the subject, the current research in quantum gravity (spin foam models) suggest to look for BF-like formulations for the Husain-Kuchar model with the hope that these can help to understand the role of the Hamiltonian constraint of general relativity at the quantum level.

In last line of thought and at the classical level, there exist a previous work by Barbero et al in which the HusainKuchar model is described as two interacting unconstrained $S O(3) B F$ theories $[7]$.

In this paper, in opposition to the viewpoint adopted in Ref. [7], we study the Husain-Kuchar model as a single constrained BF theory. In particular, we want to see the differences and similarities between this theory and general relativity when both of them are formulated in the BF Lagrangian framework and we want to distinguish one from the other theory by the way the constraints on the two-forms fields are imposed. Following this line of thought, it is quite natural to look at the various ways general relativity is formulated as a constrained BF theory $[8,09,10,11,12,13,14]$ (see also Refs. 15, 16) and, in particular, to the first of these formulations which was given by Plebanski [8] and, inspired by it, try to find a formulation for the Husain-Kuchar model.

\footnotetext{
${ }^{*}$ Electronic address: merced@fis.cinvestav.mx

†Electronic address: mquesada@fis.cinvestav.mx
} 
Throughout the paper, Greek indices $\mu, \nu, \ldots=0,1,2,3$ are spacetime indices that label the points of the spacetime $\mathscr{M}^{4}$, Latin lowercase indices $a, b, c, \ldots=1,2,3$ denote space indices. When the canonical analysis is performed $\mathscr{M}^{4}$ is assumed to have the form $\mathscr{M}^{4}=\mathbb{R} \times \Sigma$ with $\Sigma$ compact and without a boundary (to avoid boundary terms) and $\left(x^{\mu}\right)=\left(x^{0}, x^{a}\right)$ with $x^{0}$ and $x^{a}$ labeling the points along $\mathbb{R}$ and $\Sigma$, respectively.

Let us first recall the Plebanski's formulation for general relativity as a constrained BF theory, which is based on the action

$$
S[\Sigma, A, C, \rho]=\int_{\mathscr{M}^{4}}\left[\Sigma_{i} \wedge F^{i}[A]-\frac{1}{2} C_{i j} \Sigma^{i} \wedge \Sigma^{j}+\rho\left(C^{i}{ }_{i}-\Lambda\right)\right],
$$

where $A=A^{i} J_{i}$ is a connection one-form valued in the complexification of $s u(2)$ and $F=F^{i}[A] J_{i}$ with $F^{i}[A]=$ $d A^{i}+\frac{1}{2} \varepsilon^{i}{ }_{j k} A^{j} \wedge A^{k}$ is its curvature, $\Sigma=\Sigma^{i} J_{i}$ is a two-form valued in the complexication of $s u(2), J^{i}$ are the generators of the $s u(2)$ Lie algebra and satisfy the commutation relations $\left[J_{i}, J_{j}\right]=\varepsilon^{k}{ }_{i j} J_{k}$, the Lagrange multipliers $C_{i j}$ form a symmetric matrix $\left(C_{i j}\right)=\left(C_{j i}\right), \rho$ is a 4 -form field, and $\Lambda$ is the cosmological constant.

The variation of the action (2) with respect to all the independent fields involved yields the equations of motion

$$
\begin{array}{llr}
\delta A^{i}: & D \Sigma^{i}=0, & (3 \times 4=12 \text { equations }), \\
\delta \Sigma^{i}: F^{i}[A]=C^{i}{ }_{j} \Sigma^{j}, & (3 \times 6=18 \text { equations }), \\
\delta C_{i j}:-\Sigma^{i} \wedge \Sigma^{j}+2 \rho \delta^{i j}=0, & \text { (6 equations) }, \\
\delta \rho: & C^{i}{ }_{i}-\Lambda=0, & \text { (1 equation) } .
\end{array}
$$

Alternatively, Plebanski's action (2) acquires the equivalent form

$$
S[\Sigma, A, M]=\int_{\mathscr{M}^{4}}\left[\Sigma_{i} \wedge F^{i}[A]-\frac{1}{2} M_{i j} \Sigma^{i} \wedge \Sigma^{j}-\frac{\Lambda}{6} \Sigma^{i} \wedge \Sigma_{i}\right],
$$

which involves only 35 independent variables because the matrix $\left(M_{i j}\right)$ is now traceless from the very beginning. The equations of motion obtained from the action (4) are

$$
\begin{array}{llr}
\delta A^{i}: & D \Sigma^{i}=0, & (3 \times 4=12 \text { eqns }), \\
\delta \Sigma^{i}: & F^{i}[A]=M^{i}{ }_{j} \Sigma^{j}+\frac{\Lambda}{3} \Sigma^{i}, & (3 \times 6=18 \text { eqns }), \\
\delta M_{i j}: & \Sigma^{i} \wedge \Sigma^{j}-\frac{1}{3} \delta^{i j} \Sigma^{k} \wedge \Sigma_{k}=0, & (5 \text { eqns }) .
\end{array}
$$

The five equations for the $3 \times 6=18$ variables $\Sigma_{\mu \nu}^{i}$ given in (5) define a 13-dimensional manifold $\mathcal{M}^{13}$ embedded in the 18-dimensional space whose points are coordinatized by the variables $\Sigma^{i}{ }_{\mu \nu}$. This way of looking at $\mathcal{M}^{13}$ is directly related to the Lagrangian action principle (4) but there exists another, equivalent form, of looking at $\mathcal{M}^{13}$ which is useful to go straightforwardly to its Hamiltonian formulation. This second viewpoint follows from the fact that the points of $\mathcal{M}^{13}$ can be put in one-to-one correspondence with the points of a 13-dimensional manifold $\mathcal{N}^{13}$ defined by the nine equations

$$
\Sigma_{0 a}^{i}=-\frac{1}{4} \varepsilon a b c\left[N^{b} \widetilde{\pi}^{i c}+\underset{\sim}{\sim} \varepsilon^{i}{ }_{j k} \widetilde{\pi}^{j b} \widetilde{\pi}^{k c}\right]
$$

on the 22 coordinates $\Sigma_{0 a}^{i}, \tilde{\pi}^{i a}, N^{a}$, and $\underset{\sim}{N}$ that label the points of a 22-dimensional manifold. In Eq. (66), $\widetilde{\pi}^{i a}=\widetilde{\varepsilon}^{a b c} \Sigma_{b c}^{i}$ with $\widetilde{\varepsilon}^{a b c}=\widetilde{\varepsilon}^{0 a b c}$ the Levi-Civita tensor density. More precisely, the five equations for $\Sigma^{i}$ given in (5) can be seen as five linear equations for the nine variables $\Sigma_{0 a}^{i}$ and thus the general solution of the system of equations must involve four arbitrary functions, which are the densitizied lapse $\underset{\sim}{\sim}$ and shift $N^{a}$ that appear as Lagrange multipliers associated to the Hamiltonian and vector constraints, respectively (see Refs. [17, 18]).

Let us go now to the Husain-Kuchar model. From the already well-known fact that at the Hamiltonian level this model has not a Hamiltonian constraint, it follows that the analog of Eq. (6) for the Husain-Kuchar model is

$$
\Sigma_{0 a}^{i}=-\frac{1}{4} \varepsilon_{a b c} N^{b} \widetilde{\pi}^{i c} .
$$

In analogy to what (6) does for general relativity, the nine equations (77) define a 12-dimensional manifold $\mathcal{H}^{12}$ embedded in a 21-dimensional manifold whose points are coordinatized by $\Sigma_{0 a}^{i}, \widetilde{\pi}^{i a}$, and $N^{a}$. This viewpoint will be used below to go to the Hamiltonian formulation of the theory. However, to build the Lagrangian principle we are looking for, it is required to find an equivalent 12-dimensional manifold $\mathcal{F}^{12}$ suitable for the Lagrangian formulation in the sense that its definition be covariant and involves the $\Sigma^{i}$ only. Alternatively, a careful analysis of the way that the terms involving the lapse $\underset{\sim}{N}$ cancel to each other when the solution (6) for general relativity is inserted back into 
the equation $\Sigma^{i} \wedge \Sigma^{j}-\frac{1}{3} \delta^{i j} \Sigma^{k} \wedge \Sigma_{k}=0$ leads us to the covariant equation that (7) must satisfy for the Husain-Kuchar model. This equation is

$$
\Sigma^{i} \wedge \Sigma^{j}=0
$$

In fact, the six equations for the $3 \times 6=18$ variables $\Sigma^{i}{ }_{\mu \nu}$ given in (8) define the 12-dimensional manifold $\mathcal{F}^{12}$ embedded in the 18-dimensional space whose points are coordinatized by the $\Sigma^{i}{ }_{\mu \nu}$. The points of $\mathcal{F}^{12}$ can be put in one-to-one correspondence with the points of the 12-dimensional manifold $\mathcal{H}^{12}$. Equivalently, if the equation (77) is inserted back into (8), these equations are automatically satisfied.

Therefore, the constrained BF action for the Husain-Kuchar model we are proposing is, essentially, the one obtained from the Plebanski action (2) by removing the condition $C^{i}{ }_{i}-\Lambda=0$ on the Lagrange multipliers $C_{i j}$. To be precise, the action is given by

$$
S[\Sigma, A, C]=\int_{\mathscr{M}^{4}}\left[\Sigma_{i} \wedge F^{i}[A]-\frac{1}{2} C_{i j} \Sigma^{i} \wedge \Sigma^{j}\right]
$$

where there are six independent components in the symmetric matrix $\left(C_{i j}\right)$. The variation of the action (9) gives the equations of motion

$$
\begin{array}{llr}
\delta A^{i}: & D \Sigma^{i}=0, & (3 \times 4=12 \text { equations }), \\
\delta \Sigma^{i}: & F^{i}[A]=C^{i}{ }_{j} \Sigma^{j}, & (3 \times 6=18 \text { equations }), \\
\delta C_{i j}: & \Sigma^{i} \wedge \Sigma^{j}=0, & \text { ( } 6 \text { equations }),
\end{array}
$$

With the use of (7), the Hamiltonian analysis of the action (9) is straightforward. In fact, if (7) is inserted back into the action (9), it acquires the Hamiltonian form

$$
S\left[A_{a}^{i}, \widetilde{\pi}_{i}^{a}, \lambda^{i}, N^{a}\right]=\int_{\mathscr{M}^{4}} d^{4} x\left[\dot{A}_{a}^{i} \widetilde{\pi}_{i}^{a}-\lambda^{i} \widetilde{\mathcal{G}}_{i}-N^{a} \widetilde{V}_{a}\right],
$$

where

$$
\begin{aligned}
\widetilde{\mathcal{G}}_{i} & =D_{a} \widetilde{\pi}_{i}^{a} \approx 0, \\
\widetilde{V}_{a} & =F^{i}{ }_{a b} \widetilde{\pi}_{i}^{b} \approx 0,
\end{aligned}
$$

are the Gauss and vector first-class constraints corresponding to the Husain-Kuchar model [2]. Therefore, actions (1) and (9) are dual to each other in the sense that both describe the same (classical) physics but they involve different fields.

The action principle (9) is of the form $S\left[B^{M}, A^{N}\right]=\int_{\mathscr{M}^{4}} B^{M} \wedge F_{N}[A]$, where $F=F^{M}[A] X_{M}$ with $F^{M}[A]=$ $d A^{M}+\frac{1}{2} C^{M}{ }_{N L} A^{N} \wedge A^{L}$ is the curvature of a $\mathfrak{g}$-valued connection one-form $A=A^{M} X_{M}, B=B^{M} X_{M}$ is also a $\mathfrak{g}$-valued 2 -form, Latin capital indices $M, N, L, \ldots=1, \ldots, \operatorname{dim}(\mathfrak{g})$ are Lie algebra indices that are raised and lowered with respect to $k_{M N}:=k\left(X_{M}, X_{N}\right)$, which is a scalar product among the generators with respect to an inner product $k$ in the Lie algebra $\mathfrak{g}$. The inner product is assumed to be invariant under the action of the group which means that the structure constants $C^{L}{ }_{M N}$ are totally antisymmetric. The generators $X_{M}$ satisfy $\left[X_{M}, X_{N}\right]=C^{L}{ }_{M N} X_{L}$. This action principle for the particular Lie algebras $S O(3,1)$ and $S O(4)$ was reported in Ref. 19. However, its relationship with the Husain-Kuchar model was not set there. More recently, it is mentioned in Ref. 20 that (9) is general relativity with the Hamiltonian constraint removed. Nevertheless, it is not mentioned there to which of all Hamiltonian formulations of general relativity (9) is related nor how this relationship can be achieved. Moreover, the relationship of (9) to the Husain-Kuchar model is also not made there.

Thus, we have shown the equivalence between the action principle (9) and the Husain-Kuchar model (10) by performing the canonical analysis of the action (9) and showing that the result so obtained is the one that follows from the canonical analysis of the action (1). The fact that the Hamiltonian analysis of the Husain-Kuchar model can be carried out so easily is one of the advantages of the action principle (9) over the action (11). Notice that this property of the action (91) is similar to the one of the Plebanski action (2) in the sense that also the Hamiltonian analysis of the Plebanski action leads immediately to the Hamiltonian description of general relativity in terms of Ashtekar variables [17, 18], in opposition to what is usually done and that consists in performing the Dirac analysis to the self-dual Palatini action or performing a complex canonical transformation from the triad and the extrinsic curvature 1, 3, 4].

Up to here, the main results are the action principle (9) and its Hamiltonian analysis. It is possible to go further. In the same sense that the Plebanski action (2) leads to the self-dual Palatini action for general relativity introduced by Samuel and Jacobson and Smolin by solving the constraint $\Sigma^{i} \wedge \Sigma^{j}-\frac{1}{3} \delta^{i j} \Sigma^{k} \wedge \Sigma_{k}=0$ in the third line of (5) by using 
the so-called reality conditions which allows it to write $\Sigma^{i}$ in terms of a real tetrad as $\Sigma^{i}=i e^{0} \wedge e^{i}-\frac{1}{2} \varepsilon^{i}{ }_{j k} \wedge e^{j} \wedge e^{k}$ [18], it is also possible to obtain action (10) from action (9) by solving for $\Sigma^{i}$ the constraint (8) of the BF description for the Husain-Kuchar model as (modulo a constant factor) the product of the 1-forms $e^{i}$

$$
\Sigma^{i}=\varepsilon^{i}{ }_{j k} e^{j} \wedge e^{k}
$$

which amounts to parameterize the surface $\mathcal{F}^{12}$ in terms of the $3 \times 4=12$ variables $e_{\mu}^{i}$. It is clear that Eq. (8) is automatically satisfied by inserting in that equation this expression for $\Sigma^{i}$. On the other hand, by plugging (13) into the action (9), it becomes (19), as expected. Therefore, we have shown that the Lagrangian action principle for the Husain-Kuchar model can also be obtained from (9) by solving the constraint (8).

Once we know that (8) implies (13), it is natural to ask if the action principle (9) can be obtained from an BF action principle by adding to it the equation (13) with a Lagrange multiplier $\lambda^{i}$ that is a 2 -form field. By doing this, we get

$$
S[\Sigma, A, e, \lambda]=\int_{\mathscr{M}^{4}}\left[\Sigma_{i} \wedge F^{i}[A]-\lambda_{i} \wedge\left(\Sigma^{i}-\varepsilon^{i}{ }_{j k} e^{j} \wedge e^{k}\right)\right] .
$$

The variation of the action (14) with respect to the independent fields leads to the equations of motion

$$
\begin{aligned}
& \delta A^{i}: D \Sigma^{i}=0, \\
& \delta \Sigma^{i}: F^{i}[A]=\lambda^{i}, \\
& \delta \lambda^{i}: \Sigma^{i}=\varepsilon^{i}{ }_{j k} e^{j} \wedge e^{k}, \\
& \delta e^{i}: \varepsilon_{i j k} \lambda^{j} \wedge e^{k}=0 .
\end{aligned}
$$

From these it is clear that the action (11) can be independently obtained either by plugging back the expression for $\lambda^{i}$ or the expression for $\Sigma^{i}$ given in the second and third rows of (15) into the action (14). Nevertheless, it is also possible to solve for $\lambda^{i}$ by using the last equation in (15). In fact, the system of twelve linear equations for eighteen unknowns $\lambda_{\alpha \beta}^{i}$ is of rank twelve generically, which means that six out of the Lagrange multipliers $\lambda_{\alpha \beta}^{i}$ can be chosen as free parameters. Alternatively, such an equation defines a 18-dimensional manifold embedded in the space whose points are coordinatized by $\lambda_{\alpha \beta}^{i}$ and $e^{i}{ }_{\mu}$. By putting all this together, the solution for $\lambda^{i}$ acquires the form

$$
\lambda^{i}=C^{i}{ }_{j}\left(\varepsilon^{j}{ }_{m n} e^{m} \wedge e^{n}\right),
$$

which depends on eighteen independent variables: six variables encoded in the symmetric matrix $\left(C_{i j}\right)$ plus twelve involved in $e_{\mu}^{i}$, as it should be. Therefore, the last equation in (15) is automatically satisfied by plugging (16) into that equation while the remaining equations become

$$
\begin{aligned}
& D \Sigma^{i}=0, \\
& F^{i}[A]=C^{i}{ }_{j}\left(\varepsilon^{j}{ }_{m n} e^{m} \wedge e^{n}\right), \\
& \Sigma^{i}=\varepsilon^{i}{ }_{j k} e^{j} \wedge e^{k},
\end{aligned}
$$

which, using what we already have shown, namely, that the equation in the third row implies (8), the system of equations (17) becomes that given in (10), which can be obtained from (9). This way of getting (9) is a direct application of the so-called parent action method to the Husain-Kuchar action (11) [21].

In summary, the close relationship at the Hamiltonian level between the Husain-Kuchar model and general relativity expressed in terms of Ashtekar variables can be clearly appreciated when both theories are expressed at Lagrangian level as constrained BF theories. The action for the Husain-Kuchar model is the one given in (9) while general relativity is described by the Plebanski action (2). The difference between the two theories lies in the fact that the Plebanski action (2) involves one condition more than the BF-like action principle (9) for the Husain-Kuchar model: the condition on the trace of the matrix $\left(C_{i j}\right)$. At the Hamiltonian level this condition appears as one constraint more on the phase space variables: the Hamiltonian constraint, which is missing in the Hamiltonian formulation of the Husain-Kuchar model, i.e., it is also possible to say that the action for general relativity (2) is the Husain-Kuchar action supplemented with a constraint.

We conclude the paper by making some remarks and pointing out possible implications of our result:

(1) Note that if a cosmological term $\Sigma^{i} \wedge \Sigma_{i}$ is added to the action (9), the constraint (8) and its solution (7) still hold.

(2) Even though the current analysis was performed in the self-dual case, we would not expect any qualitative change between the self-dual and the real formulations, for instance, the ones obtained from the action principles 
considered in Refs. 14 and 25 by removing the condition of the trace on the Lagrange multipliers $\phi_{I J K L}$ involved in such formulations.

(3) Due to the fact the Husain-Kuchar model has not a Hamiltonian constraint, it might be possible that a quantization of the theory based in the action (9) reported in this paper (or in its Euclidean version), or in the ones suggested in item (2) using the tools of the spin foam models can help to better understand the role of the Hamiltonian constraint operator (through its absence) in the spin foam approach to the quantization of the gravitational field, by comparing these (hypothetical) spin foam models and a spin foam model for general relativity. If this could be done, such a result would be also very useful to better understand the interplay between the canonical and covariant quantizations for gravity involved in the loop quantum gravity and spin foam models approaches, respectively [22, 23] (for a spin foam quantization of the Husain-Kuchar model see Ref. 24). More precisely, our results strongly suggest that it is possible to make a slightly modification to the spin foam model for gravity considered in Ref. 25] in order to build a new one compatible with the quantum version of the constraint (8). This is linked to the item (2). The discretized version of the constraint seems to be a condition on the 4-simplex saying that it has zero volume. This is currently under investigation as well as an Euclidean version for the quantum Husain-Kuchar model and quantum gravity [26] inspired by the model considered in Ref. 27].

Warm thanks to Alejandro Perez and Carlo Rovelli for very fruitful discussions on the subject of this paper. We thank Kirill Krasnov for drawing our attention to Ref. [20]. This work was supported in part by CONACYT, Mexico, Grant Numbers 56159-F and 79629 (sabbatical term). M. Velázquez also acknowledges the financial support from CONACYT. We thank the Centre de Physique Théorique at Luminy, Marseille for all support and facilities provided for the realization of the sabbatical term of M. Montesinos and the research term of M. Velázquez.

[1] A. Ashtekar, 1986 Phys. Rev. Lett. 77, 3288 (1986).

A. Ashtekar, 1987 Phys. Rev. D 36, 1587 (1987).

A. Ashtekar, Lectures on Non-Perturbative Canonical Gravity (Notes prepared in collaboration with $R S$ Tate) (World Scientific, Singapore, 1991).

[2] V. Husain and K. Kuchar, Phys. Rev. D 42, 4070 (1990).

[3] J. Samuel, Pramana J. Phys. 28, L429 (1987).

[4] T. Jacobson and L. Smolin, Phys. Lett. B 196, 39 (1987); Class. Quantum Grav. 5, 583 (1988).

[5] J.F. Barbero G., Phys. Rev. D 51, 5498 (1995).

[6] J.F. Barbero, A. Tiemblo, and R. Tresguerres, Phys. Rev. D 57, 6104 (1998).

[7] J. Fernando Barbero G and Eduardo J.S. Villaseñor, Phys. Rev. D 63, 084021 (2001).

[8] J.F. Plebański, J. Math. Phys. 18, 2511 (1977).

[9] M. P. Reisenberger, Nucl. Phys. B 457, 643 (1995).

[10] R. Capovilla, J. Dell, and T. Jacobson, Class. Quantum Grav. 8, 59 (1991).

[11] M.P. Reisenberger, Class. Quantum Grav. 16, 1357 (1999).

[12] R. De Pietri and L. Freidel, Class. Quantum Grav. 16, 2187 (1999).

[13] D.C. Robinson, J. Math. Phys. 36, 3733 (1995).

[14] R. Capovilla, M. Montesinos, V.A. Prieto, and E. Rojas, Class. Quantum Grav. 18, L49 (2001).

[15] V. Cuesta and M. Montesinos, Phys. Rev. D 76, 104004 (2007).

[16] V. Cuesta, M. Montesinos, M. Velázquez, and J.D. Vergara, Phys. Rev. D 78, 064046 (2008).

[17] H.Y. Lee, A. Nakamichi, and T. Ueno, Phys. Rev. D 47, 1563 (1993).

[18] R. Capovilla, J. Dell, T. Jacobson, and L. Mason, Class. Quantum Grav. 8, 41 (1991).

[19] M. Montesinos, Class. Quantum Grav. 20, 3569 (2003).

[20] K. Krasnov, Mod. Phys. Lett. A 22, 3013 (2007).

[21] S. Deser and R. Jackiw, Phys. Lett. B 139, 371 (1984); Yan-Gang Miao and N. Ohta, JHEP04 (2003) 010; U. Lindström, Int. J. Mod. Phys. A 3, 2401 (1988).

[22] A. Perez, Classical Quantum Gravity 20, R43 (2003); D. Oriti, Rept. Prog. Phys. 64, 1703 (2001); J.C. Baez, Lect. Notes Phys. 543, 25 (2000); Classical Quantum Gravity 15, 1827 (1998); J. Engle, R. Pereira, and C. Rovelli, Phys. Rev. Lett. 99161301 (2007); E.R. Livine and S. Speziale, Europhys. Lett. 81 (2008) 50004.

[23] T. Thiemann, Modern Canonical Quantum General Relativity (Cambridge University Press, Cambridge, England, 2007); C. Rovelli, Quantum Gravity (Cambridge University Press, Cambridge, 2004); A. Ashtekar and J. Lewandowski, Classical Quantum Gravity 21, R53 (2004); A. Perez, "Introduction to loop quantum gravity and spin foams," Proceedings of the International Conference on Fundamental Interactions, Domingos Martins, Brazil, (2004), arXiv:gr-qc/0409061.

[24] M. Bojowald and A. Perez, "Spin Foam Quantization and Anomalies" (preprint).

[25] J. Engle, R. Pereira, and C. Rovelli, Nucl. Phys. B 798, 251 (2008).

[26] M. Montesinos and A. Perez, work in progress.

[27] M. Reisenberger, arXiv:gr-qc/9711052 v1. 